\title{
Height Dependent Motion Compensation and Coregistration for Airborne SAR Tomography
}

\author{
Matteo Nannini and Rolf Scheiber \\ German Aerospace Centre - DLR \\ Microwaves and Radar Institute \\ Oberpfaffenhofen, Germany \\ e-mail: matteo.nannini@dlr.de
}

\begin{abstract}
SAR Tomography (SARTom) is an imaging technique that allows multiple phase centers separation in the vertical (height) direction. It is performed after standard 2D SAR repeat-pass processing and operates on a stack of coregistered SAR images. Theoretically, the coregistration between two images is height dependent and the use of a reference height (or a DEM) is needed, althought not ideal in the case of volumetric target (multiple phase centers in one resolution cell). In this paper, the drawbacks related to the choice of this reference in a tomographic context are analysed and a height dependent coregistration approach is proposed. In order to do this, it is also necessary to remove processing corrections related to the reference height, such as motion compensation, and make them height dependent. The inclusion of the height dependency during the tomographic SAR processing results in a better quality of the final tomograms in terms of pseudo-power and phase centers separation. The results of the proposed approach are validated on real data acquired by the E-SAR system of the German Aerospace Centre - DLR.
\end{abstract}

\section{INTRODUCTION}

SAR tomography is an imaging technique that allows to solve for targets in the third dimension, with the help of a synthetic aperture obtained with additional observations, realized in the plane perpendicular to the flight direction. Retrieval of volume structure information (e.g. for forest classification) and the solution of layover are within the most promising applications. To demonstrate the technique, only few experimental results have been reported up to now, based on data of the airborne E-SAR system of DLR [1] and on a set of space-borne ERS data [2]. Commonly, about 10-30 acquisitions are required to perform the focusing in the third dimension (Fig.1) and generally, there is a tradeoff between the spacing between adjacent acquisitions and the total span of the tomographic aperture, as the number of acquisitions is limited [1]. The relation between the height resolution $\rho$ and the tomographic aperture dimension $L_{\text {tomo }}$ is:

$$
\rho=\frac{\lambda r_{0}}{2 L_{\text {tomo }}}
$$

where $r_{0}$ is the master slant range distance. In order to avoid ambiguities from volumetric target which height is lower than $V$, the averaged baseline $d$ must undergo:

$$
d \leq \frac{\lambda r_{0}}{2 V}
$$

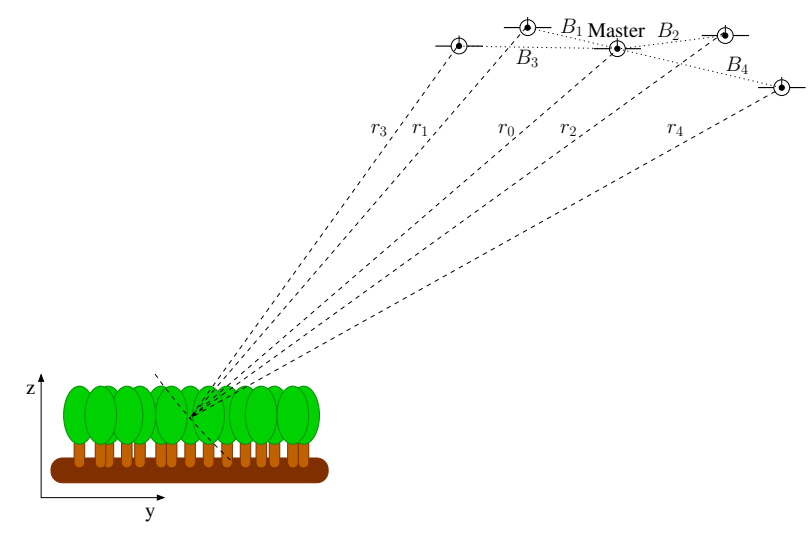

Fig. 1. Tomographic airborne constellation. The aircrafts are flying in the direction perpendicular to the depicted plane.

Now joining (1) and (2) the required number of tracks can be computed as:

$$
N=\frac{L_{\text {tomo }}}{d} .
$$

Of particular importance in the SARTom context are the data acquired most far away from the master track because they contain LOS diversity information. Considering two targets at different heights, which contribution falls in the same master resolution cell, it is possible to observe that, for large baselines, their separation in terms of slant range coordinates may become close to or even larger than one resolution cell (see Fig.2). Therefore a single height independent coregistration step can not exploit correctly the data acquired at large baselines. In addition it has to be taken into account that for standard 2D airborne SAR focusing a reference level is needed in order to perform motion compensation. The presence of this reference height level does not allow a correct focusing of targets which height is far away from it. If a target is not correctly focused, then a tomographic image will be biased for heights different from the reference one. Up to now, a standard approach used to perform tomography is done with the help of a deramping step (followed by spectral estimation techniques) that also need the definition of a reference height. If the height of the target is far away from this reference, tomographic defocusing will occur as the tomographic signal phase is not strictly quadratic. As shown recently, time domain 
beamforming can be used in order to avoid the deramping step and the drawbacks related to it [3]. Correct azimuth focusing and correct coregistration are important pre-conditions in order to apply modern beamforming techniques for tomography, that are very sensible in terms of phase errors. As was shown recently, Capon [4] and MUSIC [5] are able to improve imaging performance, e.g. to overcome the limitations posed by the Fourier resolution limit. With this work a Height dependent Motion Compensation and Coregistration (HMCC) approach for SARTom is proposed in order to compensate for the previously described effects. The results of the proposed approach are validated on real data. First a detailed description of the SARTom processing chain is given in section II. Section III describes the proposed approach and finally in section IV and $\mathrm{V}$ results and conclusion are presented.

\section{TOMOGRAPHIC SAR PROCESSING}

\section{A. SAR Processing}

In this section the SARTom pre-processing scheme is described. SARTom operates on a stack of 2D coregistered SAR images. In order to process every single SAR image the Extended Chirp Scaling (ECS) algorithm has been used [6]. Due to the deviations of the real track from the nominal one, motion compensation (moco) is required. It is usually done with respect to a reference terrain height and applies with a correction of the phase and a shift of the envelope (through an interpolation) of the image. The entity of these corrections is defined by $\Delta r\left(r_{r e f}\right)$, that is the difference between the distance real track-reference height and the nominal distance for one range azimuth $(r, a)$ position:

$$
\Delta r\left(r_{\text {ref }}\right)=r_{\text {real }}\left(r_{r e f}\right)-r_{\text {nom }}
$$

After interpolating the image according to $\Delta r\left(r_{r e f}\right)$, it is possible to apply the phase correction as:

$$
H_{\text {moco }}=\exp \left(-j \frac{4 \pi}{\lambda} \Delta r\left(r_{r e f}\right)\right) .
$$

Once that moco is carried out there are still some residual errors due to the inaccuracy of the navigation system, therefore, modern residual motion compensation techniques [7] may have to be used in order to compensate for it. Since, for estimating residual motion deviations, mis-registration in azimuth is exploited and compensated, at the end of the processing, azimuth coregistered SAR images are obtained.

At this point a range coregistration step is needed in order to align all the images for every range azimuth $(r, a)$ pair. This is done with the geometry of the scene (nominal coregistration). In order to perform the nominal coregistration a reference height (or a DEM), horizontal and vertical baseline are needed. The range coregistration function can be compute as:

$$
\Delta r=\sqrt{\left(H+B_{z}\right)^{2}+\left(\sqrt{r_{0}^{2}-H^{2}}+B_{y}\right)^{2}}-r_{0}
$$

where $H$ is the master platform height, $B_{y}$ and $B_{z}$ are the horizontal and vertical baseline respectively and $r_{0}$ is the master slant range coordinate. It is important to note that the height $H$ of the platform is defined as the distance between the platform and the reference height.

\section{B. Tomographic Processing}

After coregistration a tomographic signal $s_{t}(r, a, i)$ can be defined as:

$$
s_{t}(r, a, i)=s_{i}(r, a)
$$

where $s_{i}(r, a)$ states for the complex number related to the $i^{t h}$ SAR image at the coordinate $(r, a)$. For the sake of simplicity we will refer to $s_{t}$ as the vector containing the tomographic signal components. Bold symbols will indicate arrays.

At this point a phase calibration is required in order to align all the images with the same phase offset.

SARTom can be formulated as a DOA (Direction Of Arrival) problem. First the so-called steering vector $\boldsymbol{a}(h)$ is defined as:

$$
\boldsymbol{a}(\hat{h})=\exp \left(j \frac{4 \pi}{\lambda} \boldsymbol{R}_{\hat{h}}\right)
$$

where $\boldsymbol{R}_{\hat{h}}=\left[R_{1}(\hat{h}), \cdots, R_{N}(\hat{h})\right]$ is the array containing the distances sensors-target at a height $\hat{h}$. With the help of this vector it is possible to scan the data in the height domain. Modern beamforming methods deal with the sample complex covariance matrix $\boldsymbol{R}$. Exploitinig the statistical propriety of the data itself an enhancement of the resolution (over the Rayleigh limit [8]) and a higher side lobe suppression when comparing with the standard beamforming technique is achieved. $\boldsymbol{R}$ can be computed as:

$$
\boldsymbol{R}=\frac{1}{N} \sum_{k=-N / 2}^{N / 2} \boldsymbol{s}_{\boldsymbol{t}}(k) \boldsymbol{s}_{\boldsymbol{t}}(k)^{H} .
$$

Once that the covariance matrix has been generated Capon or MUSIC can be applied. This work will deal will Capon whose functional as a function of height is written as:

$$
\boldsymbol{C}(h)=\frac{1}{\boldsymbol{a}^{H}(h) \boldsymbol{R}^{-1} \boldsymbol{a}(h)} .
$$

Scanning now the tomographic stack in the azimuth direction a $3 D$ density reconstruction as a function of height and azimuth can be presented in a tomogram.

\section{PROPOSED APPROACH}

In order to understand and quantify the limitation of a simple motion compensation-coregistration step for SARTom, let us now consider the following geometry: a master track is located at a height of $3200 \mathrm{~m}$ with a slant range coordinate of $4200 \mathrm{~m}$ (typical values for the E-SAR system) and a slave track is separated from it by a horizontal baseline of $130 \mathrm{~m}$. If the master is imaging, in the same resolution cell, two point targets whose distance in height is $40 \mathrm{~m}$ (see Fig.2), it will imply that for the slave image the two target contributions will fall into two different resolution cells because their distances to the slave track will differ by $\cong 1.6 \mathrm{~m}$ corresponding to 1.05215 cells (it can be computed with an analogous equation as (6)). 
Therefore, it is important to align the images depending on the height that is focused at a given moment: when $P_{1}$ has to be focused the slave has to be coregistered by means of the height of $P_{1}$ itself. In this way the response of each scatterer is maximised when its height is focused. With the help of the geometry, it is possible to compute the coregistration mismatch and with an interpolation, to compensate for it.

Another thing to be noticed is that, with a fixed reference height the moco corrections will bias the focusing towards points located most close to it. The reason is twofold: on one hand because during the interpolation carried out by (4) the height dependent coregistration will not begin with the correct starting point and, on the other hand because with the phase correction (5) there will be a mismatch between the steering vector and the data.

All these considerations led to the decision to develop the HMCC algorithm for SARTom.

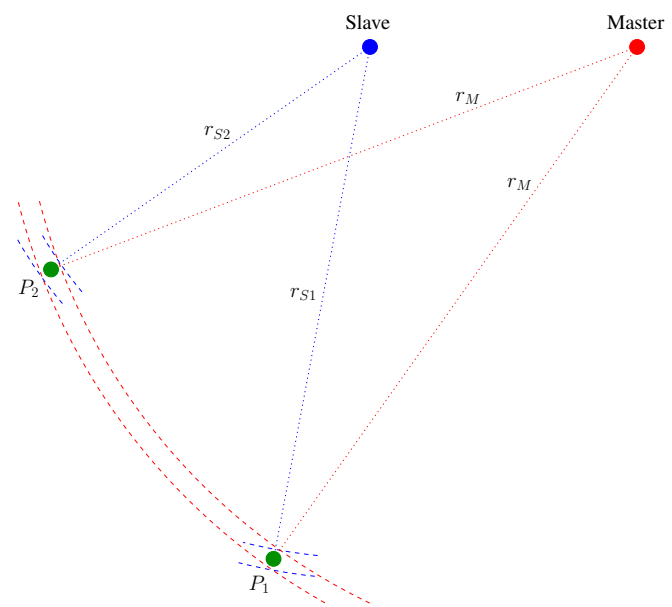

Fig. 2. SAR coregistration inaccuracy for large baseline (tomography).

\section{A. Height dependent Motion Compensation}

Theoretically, topography dependent approaches like [9] could be used in order to have an exact result of a 2D SAR processing with different heights than the reference one, but for the range of heights focused by tomography $( \pm 50 \mathrm{~m})$ a first order correction is sufficient (being aware that the envelope of the image will not change except for a subpixel shift). Another drawback related to the use of one of such a techniques for SARTom is the high time consuming impact, as this algorithm would need to be applied for all the images and for every height to be focused.

The height dependent moco approach can be divided into two parts: first it is necessary to take back the corrections done during the reference height moco and then apply new corrections as a function of the considered height. Since interpolation (4) is carried out before the phase correction (5), in order to take back these corrections and perform the socalled un-moco, it is required to perform these operations in an inverse order. Once that un-moco has been done a new moco (that will be called re-moco) can be carried out by means of
(4) and (5) considering another height $H^{\prime}$. After re-moco the height dependent coregistration can be done in an easy way because nominal tracks can be assumed and it is possible to perform only one correction for all the azimuth coordinate. A block diagram of the HMCC algorithm is represented in Fig.3.

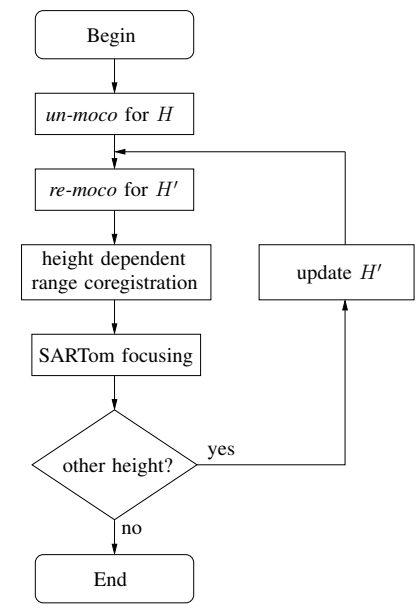

Fig. 3. Flowchart of the proposed HMCC for SARTom approach.

\section{RESUlts}

In this section results of the application of the HMCC algorithm on a tomographic dataset acquired with the E-SAR system of the Germany Aerospace Center - DLR on the test site of Oberpfaffenhofen (Germany) in May 1998, are presented. For a description of the dataset refer to [1].

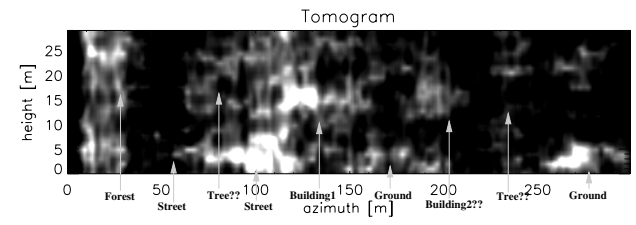

(a) Tomogram without any height dependent correction.

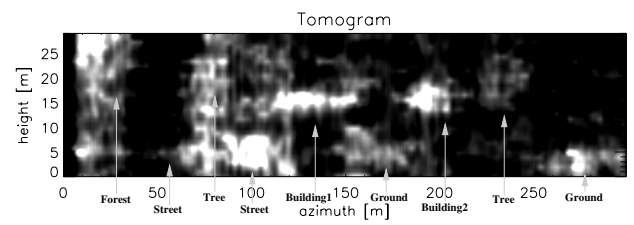

(b) HMCC tomogram.

Fig. 4. Tomograms obtained by mean of the Capon estimator.

Figure 4 represents tomograms obtained without any height dependent correction (Fig.4(a)) and with the HMCC technique (Fig.4(b)). From a first qualitative comparison it is easy to see how, in the HMCC tomogram, more features are clearly represented (e.g. a forested area, street and two buildings of the same height) with respect to the no-HMCC one.

In order to avoid basckscattering power saturation main component tomograms (mc-tomograms), obtained representing 
only the tomogram maximum for each azimuth coordinate, are represented. Figure 5 represents a comparison between mc-tomograms obtained with: fixed motion compensationcoregistration (Fig.5(a)), a height dependent motion compensation without height dependent coregistration (Fig.5(b)) and with the HMCC approach (Fig.5(c)). It is easy to check that in figure 5(a) some features are lost, indeed building 2 is not clearly focused. Probably, due to the azimuth multilook used in order to generate the sample covarinace matrix $\boldsymbol{R}$, some phase disturbances related to the presence of trees near the building itself do not allow Capon to recover the correct image. Looking now to figure 5(b) and 5(c) it is clearly possible to see how the image quality is enhanced in term of stability (the roofs of building 1-2 are rectilinear) and detection because now building 2 is clearly visible. Anyhow, it has to be noticed that the results presented in Fig.5(b) and Fig.5(c) are quite similar. This is expected because the height of the buildings is quite low (around $10 \mathrm{~m}$ ), therefore we do not expect that the height dependent coregistration plays the most important role. Anyhow, in order to check how the height dependent coregistration impacts the image quality a comparison in terms of normalised pseudo-power (estimated by the tomograms) in an azimuth interval that contains buildingl has been done (Fig.6).

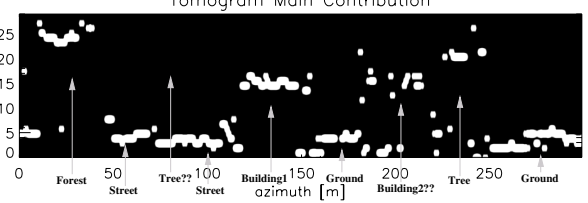

(a) No height dependent corrections.

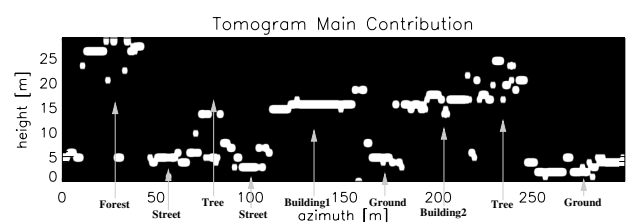

(b) Height dependent moco and no height dependent coregistration.

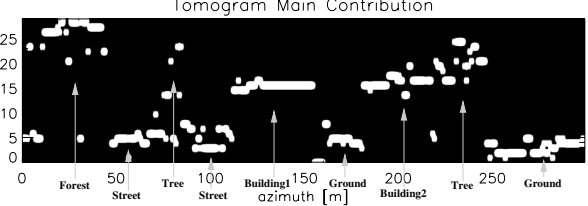

(c) HMCC.

Fig. 5. Mctomographic results.

First it has to be noticed how, the introduction of the height dependent procedures allows to achieve thinner peaks concentrating the most of the energy around the height of the building and not to spread it as done without any height dependent correction (Fig.6 dot-dashed line). As expected, due to the reduced building height, the higher quality enhancement is obtained with the height dependent moco, anyhow it is possible to see that adding the height dependent coregistration a higher power peak is achieved. We can conclude that, although the resolution with and without the height dependent coregistration remains the same, the higher peak obtained by means of the complete HMCC approach allows a better rejection of undesired components.

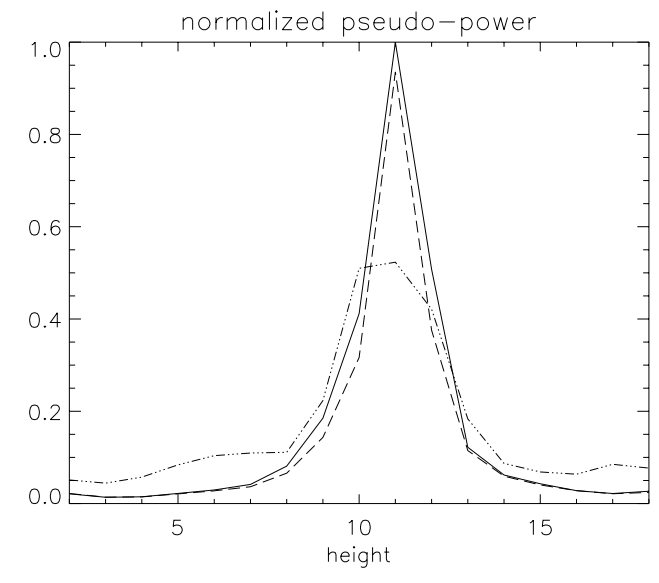

Fig. 6. Pseudo-power comparison: (dot-dashed line) no height dependent corrections, (dashed line) height dependent motion compensation and no height dependent coregistration, (solid line) HMCC.

\section{CONCLUSION}

In this paper a new approach for SAR Tomography has been proposed and validated on real data. The HMCC algorithm allows SARTom with higher quality in terms of imaging and pseudo-power separation for both man made targets and forested areas (see Fig.4). Concerning the algorithm itself, it has been shown that the height dependent coregistration enhances the performance also when the scatterers are located in a reduced range of heights. Further investigations will be carried out in the frame of a quantitative analysis on volumetric structures.

\section{REFERENCES}

[1] A. Reigber and A. Moreira, First Demonstration of Airborne SAR Tomography using Multibaseline L-Band Data, IEEE Trans. on Geosc. and Remote Sensing Vol. 38, no.5, pp.2142-2152, 2000

[2] G. Fornaro et al., Three-Dimensional Multipass SAR Focussing: Experiments with Long-term Spaceborne Data, IEEE Trans. on Geosc. and Remote Sensing, vol.43, no.4, pp.702-714, 2005

[3] M. Nannini and R. Scheiber, A Time Domain Beamforming Algorithm for SAR Tomography, Proc. EUSAR conf., Dresden, 16-18 May, 2006

[4] F. Lombardini and A. Reigber, Adaptative spectral estimation for multibaseline SAR Tomography with airborne L-band data, in Proc. IGARSS, Toulouse, France, 2003

[5] S. Guillaso and A. Reigber, Scatterer Characterisation Using Polarimetric SAR Tomography, in Proc. IGARSS, Seoul, Korea, 2005

[6] A. Moreira et al., Extended Chirp Scaling Algorithm for Air- and Spaceborne SAR Data Processing in Stripmap and ScanSAR Imaging Modes, IEEE Trans. on Geosc. and Remote Sensing Vol. 34, no.5, pp.1123 - 1136, 1996

[7] P. Prats et al., Interpolation-Free Coregistration and Phase Correction of Airborne SAR Interferograms, to be published in IEEE Geosc. and Remote Sensing Letters.

[8] P. Stoica and R. Moses, Introduction to Spectral Analysis, N.J.:Prentice Hall, 1997.

[9] P. Prats et al., Comparison of Topography- and Aperture Dependent Motion Compensation Algorithms for Airborne SAR, to be published in IEEE Geosc. and Remote Sensing Letters. 